Laurent C. Dukas

\section{Ursachen des Ulcus cruris venosum}

Die pathologischen Ursachen des venösen Ulcus cruris sind noch nicht vollständig bekannt, jedoch wird eine trophische Störung als wichtiger pathogener Faktor angesehen. Diese entsteht aufgrund einer ödembedingten Mikrozirkulationsstörung mit einer Mangelversorgung des Gewebes. Auch entzündliche Prozesse, freie Radikale und eine Mastzellaktivierung werden als Mitursachen diskutiert [2].

\section{Therapiemöglichkeiten bei Ulcus cruris venosum}

Neben der Behandlung einer Grunderkrankung steht bei venösen Beinulzera die Kompressionstherapie im Vordergrund, gefolgt von operativen Verfahren (Venenchirurgie, endovenöse Verfahren oder direkte Ulkus-chirurgische Methoden) [1]. Aufgrund seiner durchblutungsfördernden und antientzündlichen Wirkungen kommt auch das Phytotherapeutikum Padma 28 als adjuvante Behandlung infrage [3].

Padmed Circosan ${ }^{\circledR}$ steht speziell dem Arzt zur Verfügung. Es ist kassenzulässig und identisch mit Padma ${ }^{\circledR} 28$.

\title{
Padma 28 bei venösem Ulcus cruris und durch chronisch-venöse Insuffizienz bedingten Ödemen - ein Fallbericht aus der Praxis
}

Chronische, d.h. schlecht heilende, Wunden sind auch in der Allgemeinpraxis ein wichtiges Thema und können einen hohen Leidensdruck bei den Betroffenen auslösen sowie die Lebensqualität massiv beeinträchtigen. Verschiedene Grunderkrankungen können zu chronischen Wunden, d.h. Wunden, die über 3 Monate bestehen, führen, darunter die periphere arterielle Verschlusskrankheit (PAVK), die arterielle Hypertonie und der Diabetes mellitus (diabetisches Fusssyndrom) [1]. Die häufigste Form chronischer Wunden ist jedoch das Ulcus cruris venosum, bei dem die Ulzerationen an den Unterschenkeln durch eine chronisch-venöse Insuffizienz (CVI) verursacht werden [1].

\section{Wirkmechanismen von Padma 28}

Klinische Studien zeigen eine Wirksamkeit des Kombinationspräparats Padma 28 bei arteriellen Durchblutungsstörungen, vor allem bei PAVK $[4,5]$. Das Arzneimittel wird auch bei venösen Störungen, wie schwere Beine und Ödeme, eingesetzt [6, 7].

Experimentelle Studien zeigen vielfältige Wirkmechanismen, die beim Ulcus cruris venosum eine Rolle spielen könnten. Einerseits hat Padma 28 starke antientzündliche Effekte wie z.B. die Hemmung der Produktion von proinflammatorischen Zytokinen und der Aktivierung von Immunzellen [8-10]. Andererseits wirkt es auch antioxidativ und hat wundheilungsfördernde Eigenschaften $[8,11,12]$.

Im Folgenden wird ein Fall aus der Praxis beschrieben, bei dem Padma 28 als adjuvante Therapie bei CVIbedingten Ödemen und Ulzera eingesetzt wurde.

\section{Fallbericht}

Ein 69-jähriger Mann mit CVI mit Ödemen vor allem an den unteren Extremitäten, arterieller Hypertonie, Hypercholesterinämie und Status nach Kniekontusion rechts mit Differenzialdiagnose entzündliche HoffaKörper nach Trauma stellte sich vor mit ca. $5 \mathrm{~cm}$ langen, schmalen venösen Ulzera, beidseits am medialen Knöchel. Weiter hatte er seit mindestens 5 Jahren an beiden Unterschenkeln streuende, infizierte Kontaktekzeme von einer Fläche von je ca. 18 $\times 12 \mathrm{~cm}$.

Der Patient hatte Blutdrucksenker (Perindopril $4 \mathrm{mg} / \mathrm{Tag}$ ), Cholesterinsenker (Atorvastatin $10 \mathrm{mg} / \mathrm{Tag}$ ) sowie ein Vitamin-D-Präparat (Colecalciferol 2000 IE/Tag). Eine ebenfalls bestehende benigne Prostatahyperplasie wurde mit Dutasterid/Tamsulosin $0,5 / 0,4 \mathrm{mg} / \mathrm{Tag}$ behandelt.

Die Behandlung der bereits seit Jahren bestehenden Veränderungen an den unteren Extremitäten mit $10 \mathrm{mg} /$ Tag Torasemid und dem Venenmittel Naftazon (30 mg/Tag) zeigte kein be-

\section{KARGER}

(C) 2016 S. Karger GmbH, Freiburg

Fax +497614520714 


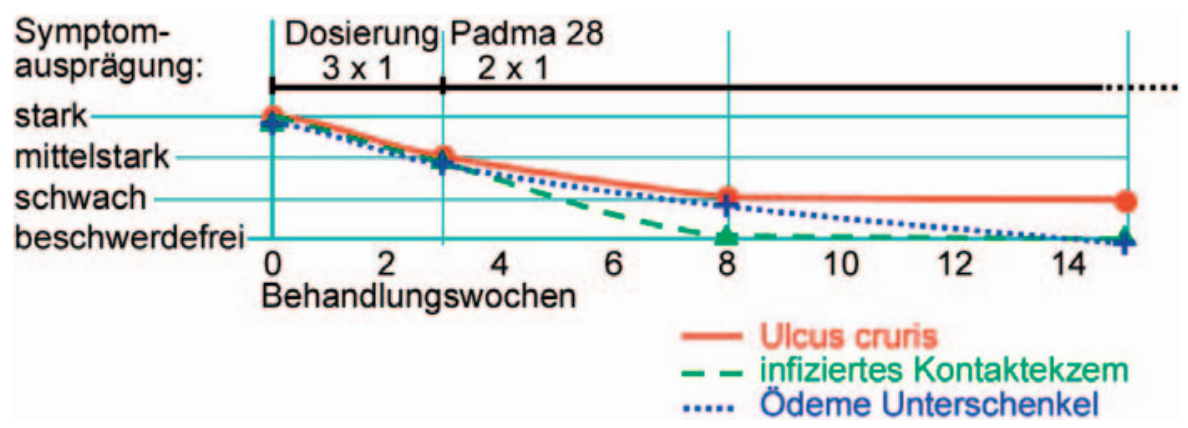

Abb. 1. Symptome, Symptomausprägung und Dosierung von Padma 28 über den Beobachtungszeitraum.

friedigendes Resultat. Ausserdem benutzte der Patient Kompressionskniestrümpfe der Klasse II. Zur lokalen Wundtherapie wurden hydropolymere Wundauflagen, Zinkpaste und eine Natriumhyaluronat-Creme angewendet. Auch ein angiochirurgischer Eingriff brachte keine Besserung.

Mit dem Ziel, die Abheilung der Ulzera durch eine verbesserte Durchblutung zu unterstützen und die Ödeme an den Unterschenkeln zu reduzieren, wurde zusätzlich zur bestehenden Therapie Padma 28 eingesetzt. Die Anfangsdosierung war 3 Kapseln täglich (1-1-1). Da sich bereits nach 3 Wochen eine deutliche Verbesserung der Ulzera, des Ekzems sowie der Ödeme einstellte (von stark ausgeprägt auf mittel), wurde die Dosis auf 2 Kapseln täglich (1-0-1) reduziert (Abb. 1). Bei der nächsten Visite nach 5 Wochen war das infizierte Kontaktekzem völlig verschwunden, und die Ödeme und
Ulzera hatten sich weiter verbessert. Nach weiteren 7 Wochen waren das Ekzem und die Ödeme gänzlich verschwunden; das Ulkus links war komplett abgeheilt und das rechts in Abheilung begriffen.

Da aufgrund der CVI und der Hypertonie ein Rezidivrisiko besteht und weil der Patient mit der Wirksamkeit und Verträglichkeit sehr zufrieden war, wurde die Behandlung mit Padma 28 in der Erhaltungsdosis $2 \times 1$ Kapsel pro Tag weitergeführt. Das Ulcus cruris rechts ist innerhalb von 24 Wochen wesentlich kleiner geworden und bis auf eine Läsion von $0,2 \times 0,4$ $\mathrm{cm}$ abgeheilt. Es befindet sich weiter in Abheilung. Die Läsionen links, welche den Patienten jahrelang belastet hatten, sind völlig abgeheilt; es sind keine Rezidive aufgetreten. Während der Behandlung traten keine Nebenwirkungen auf, und die Verträglichkeit war sehr gut.

\section{Fazit für die Praxis}

Chronische Wunden, offenes Bein oder Ulcus cruris venosum kommen auch in der Allgemeinpraxis häufig vor. Über eine Behandlung einer allfälligen Grunderkrankung, eine Kompressionstherapie und eine lokale Wundbehandlung hinaus steht in der konservativen Therapie wenig zur Verfügung bzw. wird kaum von den Fachgesellschaften empfohlen. Im chronischen Fall, bei Bestehen einer Wunde über 3 Monate und oft wie im beschriebenen Fall sogar über Jahre hatten diese Therapien aber keinen oder nur unbefriedigenden Erfolg.

Daher kann eine probatorische Therapie durchaus sinnvoll sein, sei es um zu versuchen, die Notwendigkeit eines chirurgischen Eingriffs abzuwenden, oder um einen solchen zu begleiten.

Im beschriebenen Fall konnten durch den Einsatz von Padma 28 seit Jahren bestehende, therapieresistente Unterschenkelulzera innerhalb kurzer Zeit in Abheilung gebracht und die Ödeme beseitigt werden. Wirkmechanismen könnten hierbei die durchblutungsfördernden, antientzündlichen und antioxidativen Eigenschaften des Präparats sein, die durch eine systemische Verbesserung der Durchblutung und damit des Versorgungszustands des betroffenen Gewebes die Wundheilung unterstützen.

\section{Literatur}

1 Kahle B, Hermanns HJ, Gallenkemper G: Evidence-based treatment of chronic leg ulcers. Dtsch Arztebl Int 2011;108:231-237.

2 Meissner MH, Moneta G, Burnand K, Gloviczki P, Lohr JM, Lurie F, Mattos MA, McLafferty RB, Mozes G, Rutherford RB, Padberg F, Sumner DS: The hemodynamics and diagnosis of venous disease. J Vasc Surg 2007;46(suppl):4-24.

3 Rüttgers JO: Crux medicorum: Das offene Bein. Schweiz Z Ganzheitsmed 2004;16:278-280.

4 Melzer J, Brignoli R, Diehm C, Reichling J, Do DD, Saller R: Treating intermittent claudication with Tibetan medicine Padma 28: does it work? Atherosclerosis 2006;189:39-46.

$\checkmark 5$ Vennos C, Melzer J, Saller R: Clinical studies on the efficacy and safety of Padma 28, a complex herbal formulation from Tibetan medicine: an overview. Forsch Komplementmed 2013;20(suppl 2):25-30.

6 Locher A: Padma 28 bei venösen Beinbeschwerden - ein Fallbericht. Schweiz Z Ganzheitsmed 2012;24:272-273.

7 Kneip B: Padma 28 bei Zytostatika-induzierten Dysästhesien und Ödemen der Hände ein Fall aus der Praxis. Forsch Komplementmed 2013;20(suppl 2):33-34.

$>8$ Ueberall F, Fuchs D, Vennos C: Das anti-inflammatorische Potential von Padma 28 Übersicht experimenteller Daten zur antiatherogenen Wirkung und Diskussion des Vielstoffkonzepts. Forsch Komplementärmed 2006;13(suppl 1):7-12.

-9 Barak V, Kalickman I, Halperin T, Birkenfeld S, Ginsburg I: Padma 28, a Tibetan herbal preparation is an inhibitor of inflammatory cytokine production. Eur Cytokine Netw 2004; 15:203-209.
10 Winther K, Kharazmi A, Himmelstrup H, Drabaek H, Mehlsen J: PADMA 28, a botanical compound, decreases the oxidative burst response of monocytes and improves fibrinolysis in patients with stable intermittent claudication. Fibrinolysis 1994;8(suppl 2):47-49.

11 Ginsburg I, Sadovnik M, Sallon S, et al: Padma 28, a traditional Tibetan herbal preparation inhibits the respiratory burst in human neutrophils, the killing of epithelial cells by mixtures of oxidants and pro-inflammatory agonists and peroxidation of lipids. Inflammopharmacology 1999;7:47-62.

12 Aslam MN, Warner RL, Bhagavathula N, Ginsburg I, Varani J: A multi-component herbal preparation (PADMA 28) improves structure/function of corticosteroid-treated skin, leading to improved wound healing of subsequently induced abrasion wounds in rats. Arch Dermatol Res 2010;302:669-677. 